Research Paper

\title{
A Preliminary Study on Exploring a potential Ultrasound Method for Predicting Cervical Cancer
}

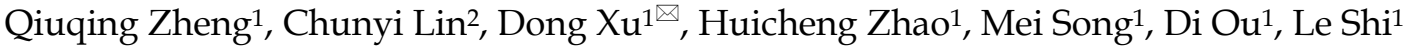 \\ 1. Department of ultrasound, The Cancer Hospital of the University of Chinese Academy of Sciences (Zhejiang Cancer Hospital), Institute of Basic Medicine \\ and Cancer (IBMC), Chinese Academy of Sciences, Hangzhou, Zhejiang, China. \\ 2. Institute of electronics and information, South China University of technology, Guangzhou, China. \\ $\square$ Corresponding author: Dong Xu, Department of ultrasound, The Cancer Hospital of the University of Chinese Academy of Sciences (Zhejiang Cancer \\ Hospital), Institute of Basic Medicine and Cancer (IBMC), Chinese Academy of Sciences, Hangzhou, Zhejiang, China. E-mail: xudong@zjcc.org.cn.
}

() The author(s). This is an open access article distributed under the terms of the Creative Commons Attribution License (https://creativecommons.org/licenses/by/4.0/). See http://ivyspring.com/terms for full terms and conditions.

Received: 2021.03.14; Accepted: 2021.12.05; Published: 2022.01.01

\begin{abstract}
Background: The level of cervical cancer screening in underdeveloped countries is far behind that of developed countries mostly because current cervical cancer screening methods are difficult to implement in underdeveloped countries. The use of non-invasive, repeatable, and low-cost ultrasound needs to be accessed.

Methods: The Canadian Sonix TOUCH ultrasound system and transvaginal ultrasound probe were used to record ultrasound radio frequency (RF) signals from cervical tissues of 69 patients with cervical cancer and 37 healthy women. The self-compiled RF time series signal analysis software was used to extract 3 different dimensions of parameters of the region of interest (ROI), including time domain, frequency domain, and fractal dimension (FD). Fourteen spectrum characteristic parameters were extracted, of which structure function method FD (SFD) and Higuchi FD belonged to FD parameters; slope, intercept, midbandfit, S1, S2, S3, and S4 were frequency domain parameters; and fuzzy entropy, kurtosis, peak, cross zero count, and cross zero standard deviation (Std) were time domain parameters.

Results: The average values of the five time-domain characteristic parameters of cervical cancer tissues were smaller than those of normal cervical tissues (fuzzy entropy: $1.70 \pm 0.29$ vs. $1.83 \pm 0.20$; kurtosis: $0.347 \pm 0.03$ vs. $0.350 \pm 0.02$; peak: $1989.9 \pm 166.8$ vs. $2024.69 \pm 187.5$; cross zero count: $3.77 \pm 0.31$ vs. $3.81 \pm 0.29$; cross zero Std: $1.26 \pm 0.17$ vs. $1.33 \pm 0.14)$, although the differences were not statistically significant $(P=0.130,0.326,0.618,0.442$, and 0.204 , respectively). The average values of the two FD characteristic parameters and the seven frequency domain characteristic parameters of cervical cancer tissues were larger than those of normal tissues (SFD: $1.84 \pm 0.28$ vs. $1.46 \pm 0.39$; Higuchi FD: $1.71 \pm 0.30$ vs. $1.28 \pm 0.30$; slope: $-0.32 \pm 0.08$ vs. $-0.26 \pm 0.05$; intercept: $0.48 \pm 0.02$ vs. $0.46 \pm 0.02$; midbandfit: $0.35 \pm 0.03$ vs. $0.33 \pm 0.03$; S1: $15.66 \pm 1.01$ vs. $13.57 \pm 1.69$; S2: $10.12 \pm 0.69$ vs. $9.32 \pm 1.27$; S3: $9.44 \pm 1.12$ vs. $8.66 \pm 1.09$; S4: $7.67 \pm 1.01$ vs. $6.43 \pm 0.65)$, and the differences were statistically significant $(P<0.05)$. No effective parameters were found to identify cervical squamous cell carcinoma tissues with different levels of differentiation $(P>0.05)$.

Conclusion: Quantitative analysis of RF time series signals based on ultrasound RF flow is expected to become a simple and non-invasive imaging method for cervical cancer diagnosis. However, whether it can be applied to the identification of early small cervical cancer lesions remains to be determined.
\end{abstract}

Key words: tissue typing, ultrasound, radio frequency time series signal, cervical cancer

\section{Introduction}

In the past decades, the incidence and death rates of cervical cancer have decreased dramatically in developed countries partly due to advances in screening methods and human papillomavirus (HPV) vaccines [1-3]. However, the situation in underdeveloped countries is different. HPV vaccines are scarce in underdeveloped countries, and although high-quality screening can compensate for this 
shortcoming, cervical cancer screening in underdeveloped countries remains unsatisfactory. The screening process for cervical cancer, which includes cervical cytology, HPV tests, colposcopy, and cervical biopsy, is complex, time-consuming, and psychologically taxing [4]. In underdeveloped countries, the distribution of medical resources is unbalanced. In economically backward areas and primary hospitals, the promotion and application of standard cervical cancer screening procedures are limited by the lack of equipment and professional technicians [5]. The occurrence of cervical cancer is related to HPV infection. Cervical cytological screening, high-risk HPV detection, and combined screening are effective clinical detection and screening methods. Among them, liquid-based thin-layer cytology (TCT) is the first choice for cervical cancer screening, as the accuracy of examination can reach $100 \%$ in theory [6]. However, medical resources are limited in economically backward areas, and gynecologists and cytologists are not well qualified for the TCT, resulting in high false negative rates and false positive rates of cervical screening. Therefore, decreasing the incidence and mortality of cervical cancer in underdeveloped countries remains an unmet goal $[2,3,7,8]$.

Developing simpler, more homogeneous, and non-invasive imaging methods is important. Ultrasonography is a flexible green imaging method associated with several advantages, such as low-cost equipment, easy to carry, no radiation, no pain, and repeatable operation. Ultrasonography allows visualization of the shape, internal structure, boundary, and blood flow of the lesion to help distinguish benign from malignant tumors. As a first-line imaging technology in various clinical fields, ultrasonography is widely used and applied to tumor screening worldwide. Ultrasound has been used for screening of liver cancer, thyroid cancer, and breast cancer among others. A national multicenter breast screening study led by Shen et al. revealed that the sensitivity and accuracy of ultrasonography are superior to those of radiography for breast screening [9]. The American College of Radiology Imaging Network breast screening study 6666 project results showed that ultrasound was more effective for screening breast invasive carcinoma than X-ray [10]. However, traditional imaging methods including ultrasound are not effective for detecting early cervical cancer lesions [11-13]. Although imaging is included in the 2018 cervical cancer National Comprehensive Cancer Network guidelines as the staging and treatment guidance standard, it is only applicable to the middle and advanced stages of the disease $[7,14]$.
Similarly, imaging methods have limited efficacy for the early diagnosis of prostate cancer [15]. Researchers have attempted to identify prostate cancer tissue by ultrasonic tissue characterization. Ultrasound RF time series analysis was considered to be one of the most effective ultrasonic tissue characterization methods, which can find subtle differences between tissues [16, 17]. Ultrasound RF time series signals have even been used to assess the subtle changes of tumors at the beginning of chemotherapy $[18,19]$. Inspired by those, we used ultrasound RF time series analysis to identify cervical cancer tissues in this study. Because of the limited experience and because there is no precedent for the use of this method in the identification of cervical cancer tissues, we screened cases of advanced cervical cancer into the study, which were quite different from normal cervical tissues and could be visualized by traditional ultrasound.

\section{Materials and Methods}

\section{Study population}

The study was conducted between March 2015 and January 2016 at Zhejiang Cancer Hospital. The study population consisted of 69 cervical carcinoma patients and 37 health controls. All enrolled cervical cancer patients were pathologically confirmed squamous cell carcinoma of the uterine cervix with nodules visualized by transvaginal ultrasound. Patients had received previous treatment, had recurrent neoplasms or had a secondary primary malignancy were excluded. 37 healthy controls were all healthy women who came to our hospital for physical examination. They had no previous cervical disease and had not undergone cervical surgery before. All subjects were able to tolerate transvaginal ultrasound and signed informed consent before enrollment. The study was approved by the Clinical Research Ethics Committee of the Zhejiang Cancer Hospital.

\section{Ultrasound data collection}

RF ultrasound data were collected using a Sonix TOUCH (Ultrasonix Medical Corp., Richmond, BC, Canada) ultrasound machine capable of recording raw RF data. A transvaginal ultrasound probe was used with the central frequency set to $6 \mathrm{MHz}$ and a depth of $10 \mathrm{~cm}$. To collect the RF time series signals, the sonographer performed a preliminary ultrasound scan to locate the cancerous nodule and then held the probe steady for $20 \mathrm{~s}$ while a computer program stored the RF data into the memory. 


\section{Parameter extraction}

In the current study, the sonographer outlined the cancerous nodule region in the B-mode ultrasound image to allow the programmer to select the cancerous ROI accurately. The ROI size was $70 \times$ $20 \mathrm{~mm}$. We used the first 256 frames of the back scattering echo RF signal to form the $1400 \mathrm{RF}$ time series, which was used to extract the tissue characterizing features. The RF time series signal analysis software developed by the team was used for quantitative analysis of the ROI [13]. The FD, frequency domain, and time domain features of the RF time series were extracted, including 14 parameters in total (Figure 1). The calculation methods for extracting all parameters are detailed in Supplementary. Among them, SFD and Higuchi FD belong to FD parameters; slope, intercept, midbandfit, S1, S2, S3, and S4 belong to frequency domain parameters; and fuzzy entropy, kurtosis, peak value, cross zero count, and cross zero Std are time domain parameters.

\section{Results analysis}

The classification results were compared with the pathologic results, which were used as the gold standard. SPSS version 22.00 statistical software was used for statistical analysis. All research data in this study were normally distributed and had uniform
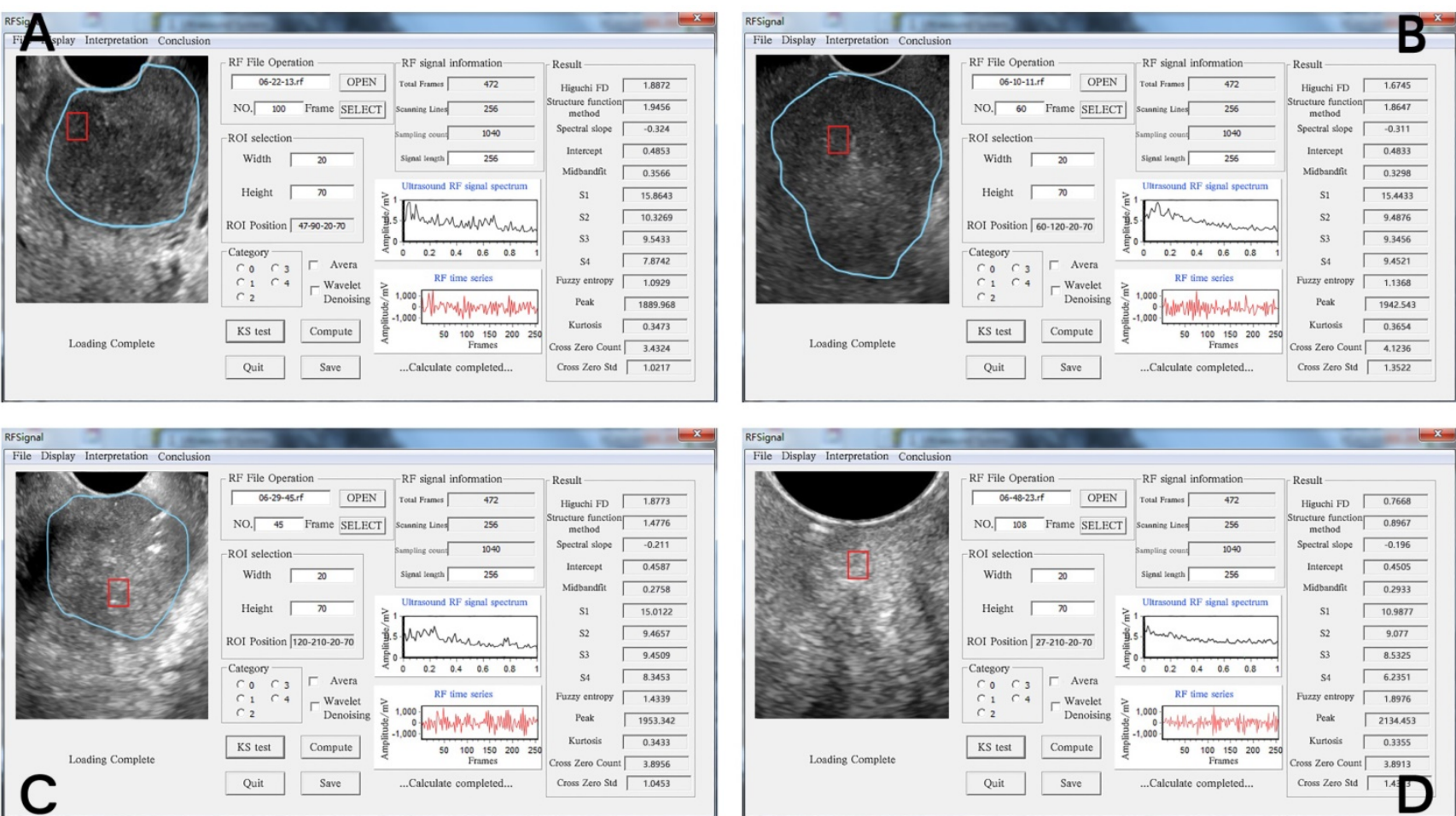

Figure 1. Operation interface for extracting RF time series parameters from cervical tissue. The four images represent the diagrams of the extraction operation interface of the RF time series parameters of different pathological types of cervical tissue. The ultrasound image of different cervical tissues is on the left of each interface; the blue line outlines the cervical cancer lesion area, and the red line outlines the ROI. The middle area of the interface is the respective RF time series spectrogram. The respective 14 RF time series parameter values are shown on the right side of the interface diagram. The pathological types of the four ROls were as follows: (A) poorly differentiated cervical squamous cell carcinoma, (B) middle-low differentiated cervical squamous cell carcinoma, (C) middle differentiated cervical squamous cell carcinoma, (D) normal cervix with a cytology negative for intraepithelial lesion or malignancy. 

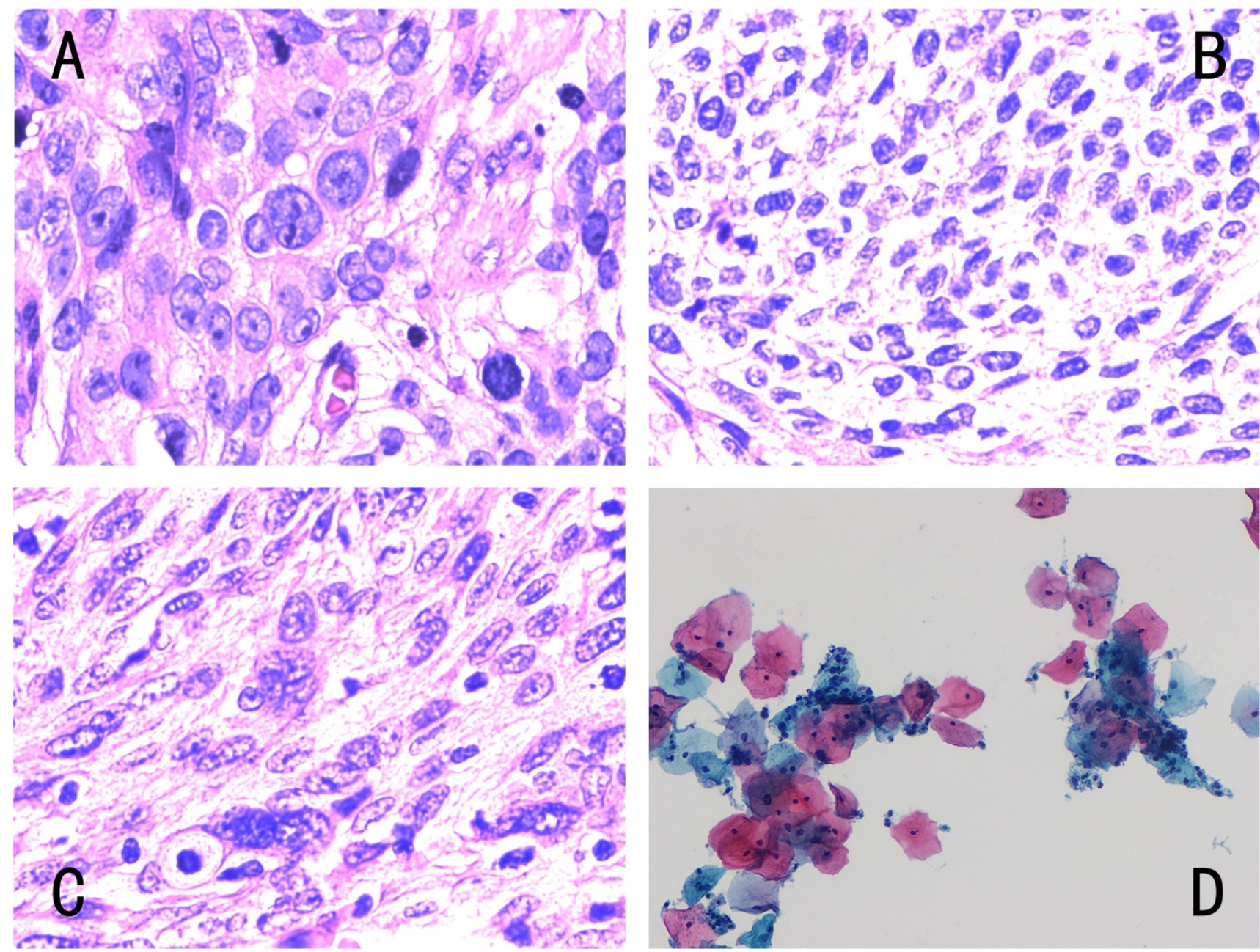

Figure 2. Different types of cervical pathology. The four images corresponded to Fig. 1, and the pathological results were as follows: (A) histopathology of low differentiated cervical squamous cell carcinoma, (B) histopathology of middle-low differentiated cervical squamous cell carcinoma, (C) histopathology of middle differentiated cervical squamous cell carcinoma, (D) cytology negative for intraepithelial lesion or malignancy. Histology was diagnosed by hematoxylin-eosin (H\&E) staining (magnification, $\mathrm{x} 400$ ) and cytology by Papanicolaou staining (magnification, $\mathrm{x} 400)$.

Table 1. Pathological Classifications of 106 subjects

\begin{tabular}{ll}
\hline Classification & Number of cases \\
\hline Low differentiated cervical SCC & 28 \\
Middle-low differentiated cervical SCC & 17 \\
Middle differentiated cervical SCC & 24 \\
NILM & 37 \\
\hline
\end{tabular}

Abbreviations: SCC, squamous cell carcinoma; NILM, negative for intraepithelial lesion or malignancy.

\section{Correlation between ultrasound RF time series features and pathological results}

A total of $14 \mathrm{RF}$ time series parameters were obtained by quantitative analysis of time series signals based on ultrasonic RF flow, all of which showed normal distribution. The mean values of FD and frequency domain parameters of cervical cancer (SFD: $1.84 \pm 0.28$, Higuchi FD: $1.71 \pm 0.30$, slope: $-0.32 \pm 0.08$, intercept: $0.48 \pm 0.02$, midbandfit: $0.35 \pm 0.03$, S1: $15.66 \pm 1.01, \quad S 2: 10.12 \pm 0.69$, S3: $9.44 \pm 1.12$, S4: $7.67 \pm 1.01)$ were higher than those of normal cervical tissues (SFD: $1.46 \pm 0.39$, Higuchi FD: $1.28 \pm 0.30$, Slope: $-0.26 \pm 0.05$, intercept: $0.46 \pm 0.02$, midbandfit: $0.33 \pm 0.03$, S1: $13.57 \pm 1.69$, S2: $9.32 \pm 1.27$, S3: $8.66 \pm 1.09$, S4: $6.43 \pm 0.65)$, and the difference was statistically significant $(P<0.05)$, On the contrary, the mean values of time domain characteristic parameters of cervical cancer (fuzzy entropy:1.70 \pm 0.29 , kurtosis: $0.347 \pm 0.03$, peak: $1989.9 \pm 166.8$, cross zero count: $3.77 \pm 0.31$, cross zero Std: $1.26 \pm 0.17$ ) were smaller than those of normal cervical tissues, but the differences were not statistically significant $(P=0.130,0.326$, $0.618,0.442$, and 0.204 , respectively) (Table 2 ).

\section{Comparison of RF time series parameters among cervical squamous cell carcinoma tissues with different degrees of differentiation}

Higher mean values of slope, intercept, S2, and S4 were correlated with a worse degree of differentiation (Table 3). Although the above parameters showed changes consistent with the changes in tissue differentiation, no statistically 
significant differences were found among three groups with different degrees of differentiation $(P>$ $0.05)$.

Table 2. Comparison of Ultrasound RF Time Series Parameters between cervical squamous cell carcinoma and normal cervical tissues

\begin{tabular}{llll}
\hline Parameter & \multicolumn{2}{l}{ Average value } & \multirow{2}{*}{$\boldsymbol{P}$ value } \\
\cline { 2 - 3 } & Tumor & Normal & $<0.001^{*}$ \\
Higuchi FD & $1.71 \pm 0.30$ & $1.28 \pm 0.30$ & $<0.001^{*}$ \\
SFD & $1.84 \pm 0.28$ & $1.46 \pm 0.39$ & $<0.001^{*}$ \\
Slope & $-0.32 \pm 0.08$ & $-0.26 \pm 0.05$ & $<0.001^{*}$ \\
Intercept & $0.48 \pm 0.02$ & $0.46 \pm 0.02$ & $0.022^{*}$ \\
Midbandfit & $0.35 \pm 0.03$ & $0.33 \pm 0.03$ & $<0.001^{*}$ \\
S1 & $15.66 \pm 1.01$ & $13.57 \pm 1.69$ & $0.001^{*}$ \\
S2 & $10.12 \pm 0.69$ & $9.32 \pm 1.27$ & $<0.001^{*}$ \\
S3 & $9.44 \pm 1.12$ & $8.66 \pm 1.09$ & $<0.001^{*}$ \\
S4 & $7.67 \pm 1.01$ & $6.43 \pm 0.65$ & 0.130 \\
Fuzzy entropy & $1.70 \pm 0.29$ & $1.83 \pm 0.20$ & 0.326 \\
Peak & $1989.9 \pm 166.8$ & $2024.69 \pm 187.5$ & 0.618 \\
Kurtosis & $0.347 \pm 0.03$ & $0.350 \pm 0.02$ & 0.442 \\
Cross Zero Count & $3.77 \pm 0.31$ & $3.81 \pm 0.29$ & 0.204 \\
Cross Zero Std & $1.26 \pm 0.17$ & $1.33 \pm 0.14$ &
\end{tabular}

$P<0.05$ indicates statistical significance;

Abbreviations: Higuchi FD, Higuchi fractal dimension; SFD, Structure function method fractal dimension; Cross Zero Std, Cross Zero Standard deviation.

Table 3. Comparison of Ultrasound RF Time Series Parameters among Different Differentiated Cervical Squamous Cell Carcinoma tissues

\begin{tabular}{|c|c|c|c|c|c|}
\hline \multirow[t]{2}{*}{ Parameter } & \multicolumn{3}{|c|}{ Cervical squamous cell carcinoma } & \multirow{2}{*}{$\begin{array}{l}F \\
\text { value }\end{array}$} & \multirow{2}{*}{$\begin{array}{l}\boldsymbol{P} \\
\text { value }\end{array}$} \\
\hline & $\begin{array}{l}\text { Low } \\
\text { differentiated } \\
\text { carcinoma }\end{array}$ & $\begin{array}{l}\text { Middle-low } \\
\text { differentiated } \\
\text { carcinoma }\end{array}$ & $\begin{array}{l}\text { Middle } \\
\text { differentiated } \\
\text { carcinoma }\end{array}$ & & \\
\hline Higuchi FD & $1.861 \pm 0.31$ & $1.87 \pm 0.39$ & $1.796 \pm 0.19$ & 0.376 & 0.688 \\
\hline SFD & $1.681 \pm 0.32$ & $1.707 \pm 0.39$ & $1.754 \pm 0.939$ & 0.468 & 0.628 \\
\hline Slope & $-0.325 \pm 0.03$ & $-0.320 \pm 0.08$ & $-0.302 \pm 0.09$ & 0.577 & 0.564 \\
\hline Intercept & $0.483 \pm 0.18$ & $0.481 \pm 0.19$ & $0.478 \pm 0.18$ & 0.369 & 0.693 \\
\hline Midbandfit & $0.353 \pm 0.03$ & $0.360 \pm 0.03$ & $0.342 \pm 0.03$ & 1.942 & 0.151 \\
\hline S1 & $15.621 \pm 0.99$ & $15.837 \pm 1.19$ & $15.587 \pm 0.93$ & 0.337 & 0.715 \\
\hline S2 & $10.246 \pm 0.63$ & $10.183 \pm 0.49$ & $9.589 \pm 0.84$ & 1.129 & 0.33 \\
\hline S3 & $9.689 \pm 0.73$ & $8.919 \pm 1.34$ & $9.52 \pm 1.25$ & 2.694 & 0.075 \\
\hline S4 & $7.747 \pm 0.87$ & $7.623 \pm 1.08$ & $7.602 \pm 1.01$ & 0.148 & 0.862 \\
\hline Fuzzy entropy & $1.763 \pm 0.23$ & $1.625 \pm 0.29$ & $1.616 \pm 0.32$ & 1.817 & 0.171 \\
\hline Peak & $1990.1 \pm 199.8$ & $2065.6 \pm 195.8$ & $1935.9 \pm 185.3$ & 2.602 & 0.074 \\
\hline Kurtosis & $0.352 \pm 0.03$ & $0.345 \pm 0.03$ & $0.342 \pm 0.03$ & 0.62 & 0.541 \\
\hline $\begin{array}{l}\text { Cross Zero } \\
\text { Count }\end{array}$ & $3.720 \pm 0.33$ & $3.739 \pm 0.31$ & $3.822 \pm 0.30$ & 0.808 & 0.45 \\
\hline Cross Zero Std & $1.279 \pm 0.17$ & $1.2321 \pm 0.18$ & $1.253 \pm 0.17$ & 0.418 & 0.66 \\
\hline
\end{tabular}

$P<0.05$ indicates statistical significance;

Abbreviations: Higuchi FD, Higuchi fractal dimension; SFD, Structure function method fractal dimension; Cross Zero Std, Cross Zero Standard deviation.

\section{Discussion}

Cervical cancer is the fourth most common cancer among women in the world, and its prognosis and survival are closely related to the stage of the disease [1]. The 5-year survival rate for localized cervical cancer is $91.5 \%$, while for late metastases it drops to $16.5 \%$ [20]. In developed countries, due to the popularity of HPV vaccine and cervical cancer screening, encouraging results have been shown in reducing the morbidity and mortality of cervical cancer patients. However, in underdeveloped countries due to the lack of medical resources, women cannot get effective early diagnosis. Most of them are already in the advanced stage when they are discovered, often accompanied by uncomfortable clinical symptoms such as abnormal vaginal bleeding. This makes standard treatment strategies ineffective, which not only affecting prognosis, but also disturbing with their normal lives $[2,4,6]$. Therefore, it is very important to make it easier for women in underdeveloped countries to get early detection of cervical cancer.

Ultrasound examination is a convenient and effective method for clinical diagnosis and treatment guidance in many diseases because it does not expose the patient to radiation, it is non-invasive, can be easily repeated, and it is cost-effective. Traditional gray-scale ultrasound imaging uses the ultrasound probe to receive the envelope amplitude of the RF echo signal. This signal processing ignores some information in the original RF signal stream, such as the frequency spectrum, phase, and the interaction between soft tissue and ultrasound. It can distinguish tissue structures with a diameter larger than the wavelength, and cannot evaluate the microstructure characteristics of tissues with a diameter smaller than the wavelength [21]. Therefore, ultrasound imaging has several shortcomings in clinical practice, such as its inability to identify certain tumor lesions, especially early small lesions. In recent years, many studies have investigated methods to extract the information related to the tissue microstructure in the original ultrasound RF signal to characterize the tissue microstructure. Among them, ultrasonic RF time series signal analysis is a widely used and reliable method. The early application of this technology in the field of cancer was limited to the identification of tumor tissues, such as prostate cancer and breast cancer [16, 17, 22]. Later studies used the same organizational characterization method to observe the response to treatment. Imani et al. used this method to distinguish ablated from non-ablated liver tissues [23], whereas $\mathrm{Li}$ et al. used the method to identify chemotherapy responders and nonresponders in preclinical breast cancer models [19]. These studies demonstrated that the RF time series analysis method is capable of characterizing the subtle differences between different tissues.

In this study, cervical cancer and normal cervical tissues were distinguished by analyzing the ultrasound RF time series signals of the microstructure of the lesion. We extracted features from three dimensions including time domain, frequency domain, and FD, and 14 parameters were extracted in 
total. Among them, the average values of the five time domain parameters of cervical cancer were smaller than those of normal tissues. The larger the value of the time domain parameters, the more uniform the tissue distribution [24, 25]. However, the differences were not statistically significant. Studies show that when performing time domain analysis of RF signals, finding the same time domain parameters of some signals does not indicate that the signals are exactly the same. The signal not only changes with time, but it is also related to information such as frequency and phase. Time domain functions can be transformed into frequency domain functions by Fourier or Laplace transformation [26-28]. According to this method, we further extracted nine parameters of frequency domain and FD. The mean values of these nine parameters in cervical cancer and normal cervical tissues were significantly different, which is consistent with previous studies. Moradi et al. found that six frequency domain parameters including slope, intercept, S1-4, and FD have good sensitivity and specificity for identifying prostate cancer [16]. Analysis of six different animal tissues showed that the six frequency domain parameters and FD have high accuracy, and FD has the highest accuracy (74\%-90\%) [29]. Uniyal et al. reclassified suspected breast cancer tissues, and found that frequency domain and FD parameters played an important role; the AUC values were estimated at $0.82(95 \% \mathrm{CI}$ : 0.63-1.00) using support vector machines and 0.69 (95\% CI: 0.47-0.91) using Random Forests [22].

In this study, we further compared the ultrasound RF time series signal parameters of cervical squamous cell carcinoma tissues with different levels of differentiation. Because we screened and enrolled all cases of cervical cancer that were visualized by grayscale ultrasound, they were all in the middle and late stages of the tumor and poorly differentiated; therefore, no cases of well-differentiated cancer were detected in this study. Among 28 cases of poorly differentiated cervical squamous cell carcinoma, 17 cases of moderate-poorly differentiated squamous cell carcinoma, and 24 cases of moderately differentiated squamous cell carcinoma tissues, the four parameters slope, intercept, S2, and S4 varied in a pattern consistent with the changes in tissue microstructure. However, the differences were not statistically significant $(P>0.05)$. This may be related to the small number of cases enrolled and the small differences between the tissues of the enrolled cases.

Frequency domain and FD of ultrasonic RF time series signals are parameters that reflect the complexity, roughness, and irregularity of the tissue surface, which may overlap with traditional gray-scale ultrasound imaging in identifying tissues.
In this study, the use of ultrasonic RF time series signals to identify cervical cancer tissue was a preliminary and simple study. The objects selected were all cervical cancer tissues that can be visualized by traditional gray-scale ultrasound. Whether this method is suitable for the early diagnosis of cervical cancer remains unknown, and further in-depth studies will be performed in the future, such as using small cervical cancer lesions as ROI. These lesions can only be identified by contrast ultrasound, but not gray-scale ultrasound. We hope that additional efforts will result in the use of this technology to identify early cervical cancer, and ultimately assist in the screening of cervical cancer.

\section{Conclusion}

Quantitative analysis of ultrasound RF time series parameters can be used for the identification of cervical cancer; however, its feasibility for the identification of cervical cancer tissues with different levels of differentiation has not been demonstrated, and whether it can be applied to early cervical cancer tissues that cannot be identified by gray-scale ultrasound remains unknown. This preliminary study showed that this method can be applied to the identification of cervical cancer, thereby providing a potential new ultrasound-based method for the diagnosis of cervical cancer. Additional studies are necessary to promote the application of ultrasound to the early diagnosis and screening of cervical cancer in future.

\section{Supplementary Material}

Supplementary information. https://www.jcancer.org/v13p0793s1.pdf

\section{Acknowledgements}

This work was supported by National Natural Science Foundation of China (No. 82071946), the Medical Health Science and Technology Project of Zhejiang Province (NO. 2021KY592).

\section{Competing Interests}

The authors have declared that no competing interest exists.

\section{References}

1. Sung H, Ferlay J, Siegel RL, Laversanne M, Soerjomataram I, Jemal A, et al. Global Cancer Statistics 2020: GLOBOCAN Estimates of Incidence and Mortality Worldwide for 36 Cancers in 185 Countries. CA: a cancer journal for clinicians. 2021; 71: 209-49.

2. Sawaya GF, Smith-McCune K, Kuppermann M. Cervical Cancer Screening: More Choices in 2019. Jama. 2019; 321: 2018-9.

3. Constantinou P, Dray-Spira R, Menvielle G. Cervical and breast cancer screening participation for women with chronic conditions in France: results from a national health survey. BMC cancer. 2016; 16: 255.

4. Wentzensen N, Clarke MA. Cervical Cancer Screening-Past, Present, and Future. Cancer epidemiology, biomarkers \& prevention: a publication of the 
American Association for Cancer Research, cosponsored by the American Society of Preventive Oncology. 2021; 30: 432-4.

5. Thekkek N, Richards-Kortum R. Optical imaging for cervical cancer detection: solutions for a continuing global problem. Nature reviews Cancer. 2008; 8: 725-31.

6. Chin FHX, Wrede CDH, Richards A, Steele A, Vicario E, McNally OM, et al. Primary HPV cervical screening: Clinical audit of outcomes of women seen at a tertiary referral centre for colposcopy in Australia. The Australian \& New Zealand journal of obstetrics \& gynaecology. 2021.

7. Devine C, Viswanathan C, Faria S, Marcal L, Sagebiel TL. Imaging and Staging of Cervical Cancer. Seminars in ultrasound, CT, and MR. 2019; 40: 280-6.

8. Girianelli VR, Thuler LC, Azevedo e Silva G. Predictive Capability of HPV and Pap Tests in Screening for Cervical Cancer over a Three-Year Follow-up. Revista brasileira de ginecologia e obstetricia : revista da Federacao Brasileira das Sociedades de Ginecologia e Obstetricia. 2016; 38: 147-53.

9. Shen S, Zhou Y, Xu Y, Zhang B, Duan X, Huang R, et al. A multi-centre randomised trial comparing ultrasound vs mammography for screening breast cancer in high-risk Chinese women. British journal of cancer. 2015; 112: 998-1004

10. Berg WA, Blume JD, Cormack JB, Mendelson EB, Lehrer D, Bohm-Velez M, et al. Combined screening with ultrasound and mammography vs mammography alone in women at elevated risk of breast cancer. Jama. 2008; 299: 2151-63.

11. Bolla D, In-Albon S, Papadia A, Di Naro E, Gasparri ML, Mueller MM, et al. Doppler Ultrasound Flow Evaluation of the Uterine Arteries Significantly Correlates with Tumor Size in Cervical Cancer Patients. Annals of surgical oncology. 2015; 22 Suppl 3: S959-63.

12. Tepper R, Zalel Y, Altaras M, Ben-Baruch G, Beyth Y. Transvaginal color Doppler ultrasound in the assessment of invasive cervical carcinoma. Gynecologic oncology. 1996; 60: 26-9.

13. Alcazar JL, Castillo G, Jurado M, Lopez-Garcia G. Intratumoral blood flow in cervical cancer as assessed by transvaginal color doppler ultrasonography: Correlation with tumor characteristics. International journal of gynecological cancer: official journal of the International Gynecological Cancer Society. 2003; 13: $510-4$

14. Bhatla N, Berek JS, Cuello Fredes M, Denny LA, Grenman S, Karunaratne K, et al. Revised FIGO staging for carcinoma of the cervix uteri. International journal of gynaecology and obstetrics: the official organ of the International Federation of Gynaecology and Obstetrics. 2019; 145: 129-35.

15. Inahara M, Suzuki H, Nakamachi H, Kamiya N, Shimbo M, Komiya A, et al. Clinical evaluation of transrectal power doppler imaging in the detection of prostate cancer. International urology and nephrology. 2004; 36: 175-80.

16. Moradi M, Mousavi P, Abolmaesumi P. Computer-aided diagnosis of prostate cancer with emphasis on ultrasound-based approaches: a review. Ultrasound in medicine \& biology. 2007; 33: 1010-28.

17. Imani F, Abolmaesumi P, Gibson E, Khojaste A, Gaed M, Moussa M, et al. Computer-Aided Prostate Cancer Detection Using Ultrasound RF Time Series: In vivo Feasibility Study. IEEE transactions on medical imaging. 2015; 34: 2248-57.

18. Lin Q, Wang J, Li Q, Lin C, Guo Z, Zheng W, et al. Ultrasonic RF time series for early assessment of the tumor response to chemotherapy. Oncotarget. 2018; 9: 2668-77.

19. Li F, Huang Y, Wang J, Lin C, Li Q, Zheng X, et al. Early differentiating between the chemotherapy responders and nonresponders: preliminary results with ultrasonic spectrum analysis of the RF time series in preclinical breast cancer models. Cancer imaging : the official publication of the International Cancer Imaging Society. 2019; 19: 61.

20. Ferlay J, Steliarova-Foucher E, Lortet-Tieulent J, Rosso S, Coebergh JW, Comber $\mathrm{H}$, et al. Cancer incidence and mortality patterns in Europe: estimates for 40 countries in 2012. European journal of cancer. 2013; 49: 1374-403.

21. Lizzi FL, Greenebaum M, Feleppa EJ, Elbaum M, Coleman DJ. Theoretical framework for spectrum analysis in ultrasonic tissue characterization. The Journal of the Acoustical Society of America. 1983; 73: 1366-73.

22. Uniyal N, Eskandari H, Abolmaesumi P, Sojoudi S, Gordon P, Warren L, et al. Ultrasound RF time series for classification of breast lesions. IEEE transactions on medical imaging. 2015; 34: 652-61.

23. Imani F, Wu MZ, Lasso A, Burdette EC, Daoud M, Fitchinger G, et al. Monitoring of tissue ablation using time series of ultrasound RF data. Medical image computing and computer-assisted intervention : MICCAI International Conference on Medical Image Computing and Computer-Assisted Intervention. 2011; 14: 379-86.

24. Ling W, Quan J, Lin J, Qiu T, Li J, Lu Q, et al. Grading fatty liver by ultrasound time-domain radiofrequency signal analysis: an in vivo study of rats. Experimental animals. 2018; 67: 249-57.

25. Moran CM, Sutherland GR, Anderson T, Riemersma RA, McDicken WN. A comparison of methods used to calculate ultrasonic myocardial backscatter in the time domain. Ultrasound in medicine \& biology. 1994; 20: 543-50.

26. Rijsterborgh H, Mastik F, Lancee CT, Verdouw P, Roelandt J, Bom N. Ultrasound myocardial integrated backscatter signal processing: frequency domain versus time domain. Ultrasound in medicine \& biology. 1993; 19: 211-9

27. Erlov T, Jansson T, Persson HW, Cinthio M. Scatterer size estimation using the center frequency assessed from ultrasound time domain data. The Journal of the Acoustical Society of America. 2016; 140: 2352.
28. Shimokado A, Kubo $\mathrm{T}$, Matsuo $\mathrm{Y}$, Ino $\mathrm{Y}$, Shiono $\mathrm{Y}$, Shimamura $\mathrm{K}$, et al. Imaging assessment and accuracy in coronary artery autopsy: comparison of frequency-domain optical coherence tomography with intravascular ultrasound and histology. The international journal of cardiovascular imaging. 2019; 35: 1785-90.

29. Moradi M, Abolmaesumi P, Mousavi P. Tissue typing using ultrasound RF time series: experiments with animal tissue samples. Medical physics. 2010; 37: 4401-13. 